\title{
INFLUENCE OF CORTISONE AND PREDNISOLONE ON HYPERBILIRUBINEMIA
}

\author{
BY RICARDO KATZ, HECTOR DUCCI, AND HERNAN ALESSANDRI \\ (From the Department of Medicine, University of Chile Medical School, Hospital Salvador, \\ Santiago, Chile)
}

(Submitted for publication November 26, 1956; accepted May 16, 1957)

Our experience (1), as well as that of others $(2-4)$, in the treatment of acute hepatitis with steroids, has shown as the most striking effect a rapid drop of serum bilirubin. This is accompanied by the clearing of clinical jaundice.

Solem and Olsen (5) have studied the influence of ACTH on the icteric index in cases of hepatitis and of post-hepatic jaundice. In the former, it dropped very rapidly but in the latter it was not significantly influenced. They believed that the different response of both types of jaundice could be used in the differential diagnosis.

Sborov, Giges, Plough, and Mandel (6) report a case of post-hepatic jaundice in which ACTH did not induce a lowering of hyperbilirubinemia.

Johnson and Doenges (7), in two cases of hepatitis with hepatocanalicular jaundice, observed a rapid drop of serum bilirubin following the administration of ACTH. In one of these patients who had a common duct drainage the drop was concomitant with the reestablishment of biliary flow. Although they do not report results in cases of post-hepatic jaundice, they suggest that the hormone could be useful in differential diagnosis as it would not influence hyperbilirubinemia in this type of icterus.

According to Patterson, Dingman, Schwachman, and Thorn (8), cortisone has a choleretic action which could explain the lowering of the serum bilirubin values.

Other investigators (4) include the increase in biliary flow among the factors which could explain the drop of hyperbilirubinemia in cases of hepatitis.

Our findings in the first case of post-hepatic jaundice who received cortisone prompted us to study the influence of steroids in cases of complete biliary obstruction. This patient showed a clearcut drop of serum bilirubin in two periods in which he received cortisone, despite a carcinoma

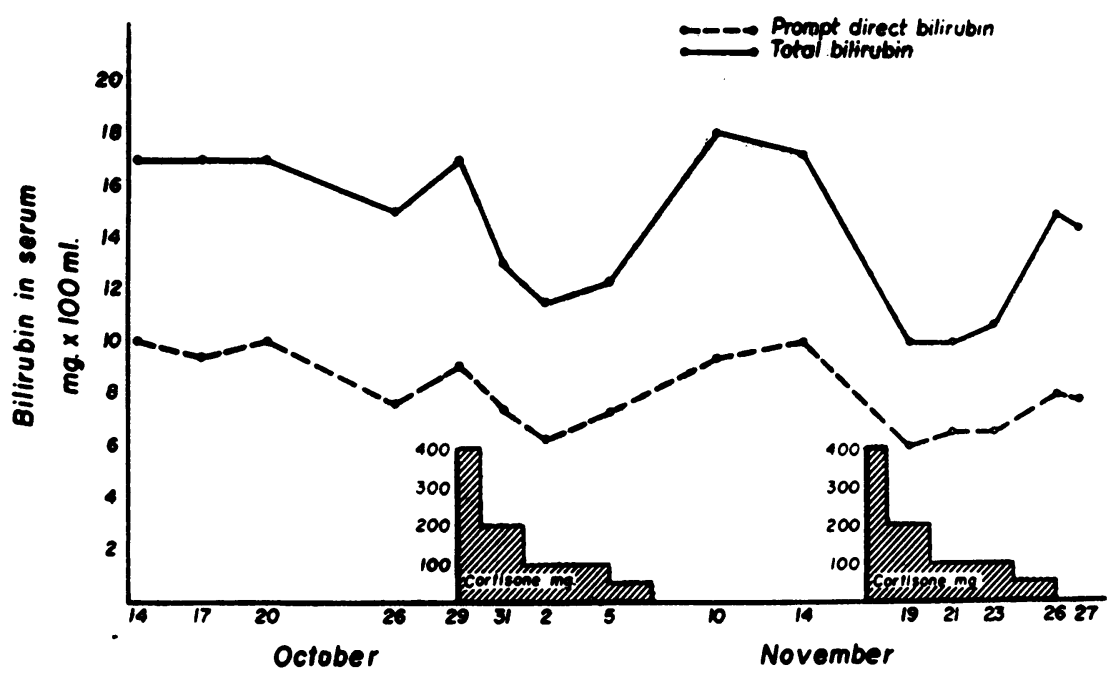

Fig. 1. Influence of Cortisone on Serum Bilirubin in Post-Hepatic Jaundice with Complete Obstruction Due to Carcinoma of the Head of the Pancreas Case 1. 38 year-old male. Hospital number 53/15891. 


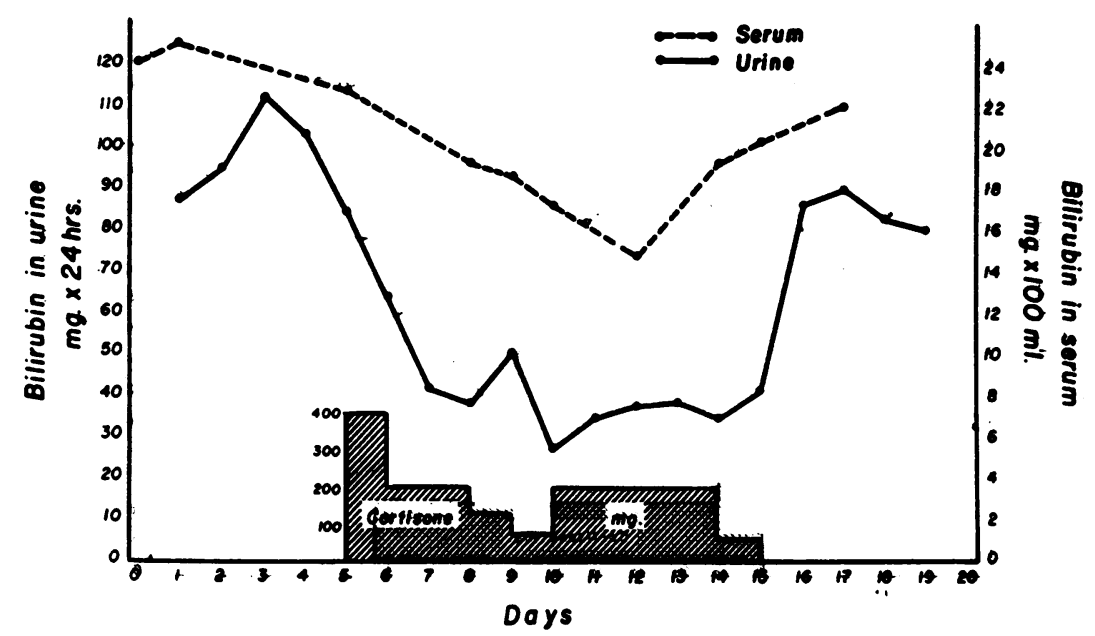

Fig. 2. Influence of Cortisone on Serum and Urine Bilirubins

Case 2. R.E., 68 year-old female. Hospital number 56/4746.

The values for urine bilirubin in this and the following figures are those obtained by Jendrassik and Gróf's method (15).

of the head of the pancreas with complete obstruction of the common bile duct, as shown at operation (Figure 1).

These results suggested that the choleretic effect, if present, was not an important factor in the reduction of serum bilirubin values. Moreover they showed that jaundice, even when due to biliary obstruction, was influenced by cortisone.

Chalmers and associates (9) have recently reported their findings with a four-day ACTH test in the differential diagnosis of jaundice. They ob- served a definite overlapping of effects, thereby failing to confirm the usefulness of the test. Among their patients with biliary obstruction, two showed more than a one-third drop of serum bilirubin despite no detectable urinary urobilinogen throughout the observation period. It is interesting to call attention, in relation to what will be reported later, to the observations of these investigators in chronic hyperbilirubinemia. They found that the drop in bilitubin in the majority of patients was presumably not a result of increased

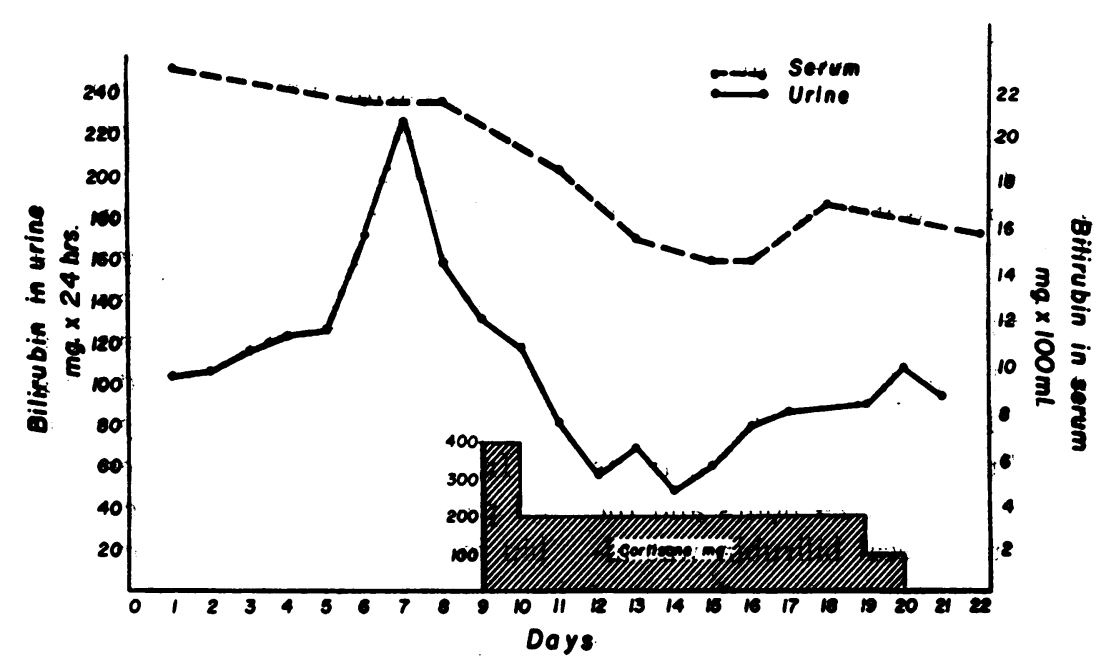

Fig. 3. Imtrotexces of Cortisone on Serum and U'rine Bilirubins

Case 3. L.S., 57 year-old male. Hospital number 56/9226. 


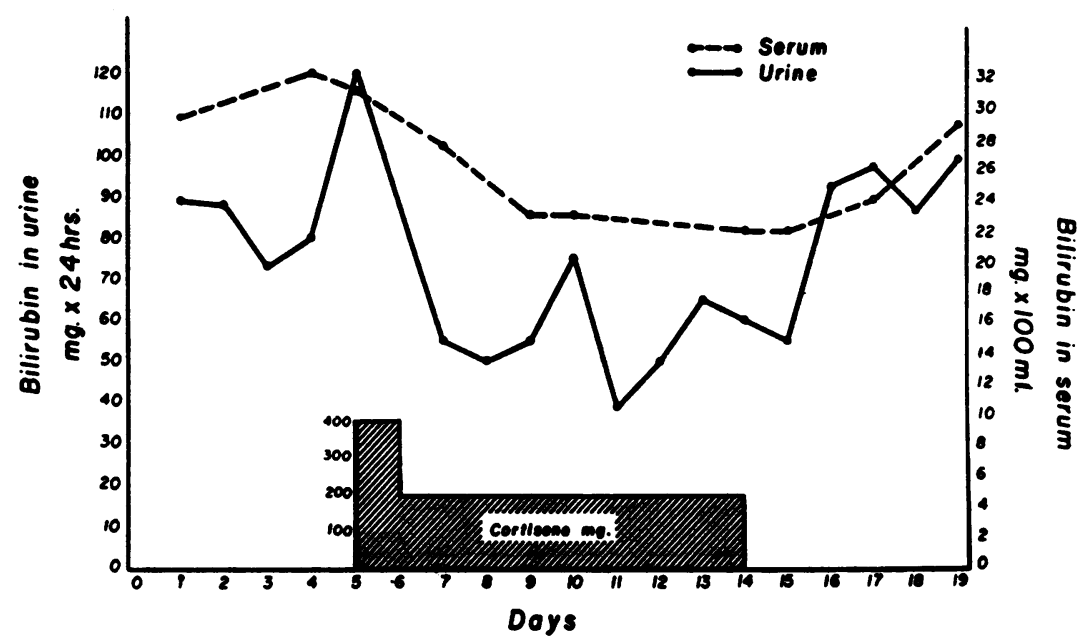

Fig. 4. Influence of Cortisone on Serum and Urine Bilirubins

Case 4. E.C., 63 year-old female. Hospital number 56/8648.

choleresis, because it was accompanied by a decrease rather than an increase in fecal urobilinogen output.

\section{METHODS OF STUDY}

For the purpose of investigating the effect of steroids on hyperbilirubinemia of cases in which choleresis could not have a role, we chose cases of post-hepatic ${ }^{1}$ jaundice with complete biliary obstruction. These patients were selected because they had sustained fecal acholia (absence of fecal urobilinogen) due to complete biliary obstruction. Three received cortisone and one prednisolone by the oral route for periods of 9 to 10 days. The dosage is indicated in Figures 2 through 5.

During the basal observation period, during the administration of the steroid, and following its withdrawal, the following laboratory tests were carried out serially: prompt direct and total serum bilirubin $(10,11)$, fecal and urinary urobilinogen $(12,13)$, urinary bilirubin with Malloy and Evelyn's (14) and Jendrassik and Gróf's (15) methods, and the qualitative investigation of fecal bilirubin (application of Watson and Hawkinson's method (16) to feces extract).

\section{RESULTS}

The results obtained are shown graphically in Figures 2 through 5. During the whole period of study the fecal urobilinogen in the four patients was constantly below $1 \mathrm{mg}$. per 24 hours and the qualitative investigation of fecal bilirubin was always negative.

It can be easily observed that cortisone as well as prednisolone, given in doses similar to those

\footnotetext{
1 Obstructive jaundice of other classifications.
}

used by the authors in the treatment of acute benign hepatitis, produced maximal drops of serum bilirubin of 28 to 34 per cent from the initial value. This drop, although evident, is less than that observed by the present investigators in acute hepatitis during the first four days of administration of cortisone ( 55.7 per cent).

The drop of serum bilirubin in our cases of complete biliary obstruction was accompanied by persistence of acholia and, in contrast to what would be expected, by a reduction of the urinary excretion of bilirubin. This reduction, although variable, was observed in all four cases during the administration of the drug. It was shown by the two methods used which gave almost identical values (urine preserved with sodium carbonate and collected under petroleum ether was used throughout).

After the withdrawal of the steroid the serum bilirubin increased, reaching pre-treatment levels in three cases within four days. In the other patient (Case No. 3) bilirubin did not reach the basal values; this patient had an important gastrointestinal hemorrhage during this period.

In addition to the changes in serum bilirubin, all patients showed an increase in urinary bilirubin following discontinuation of the steroid.

\section{DISCUSSION}

The results here reported demonstrate the effect of cortisone and prednisolone on the hyperbilirubi- 


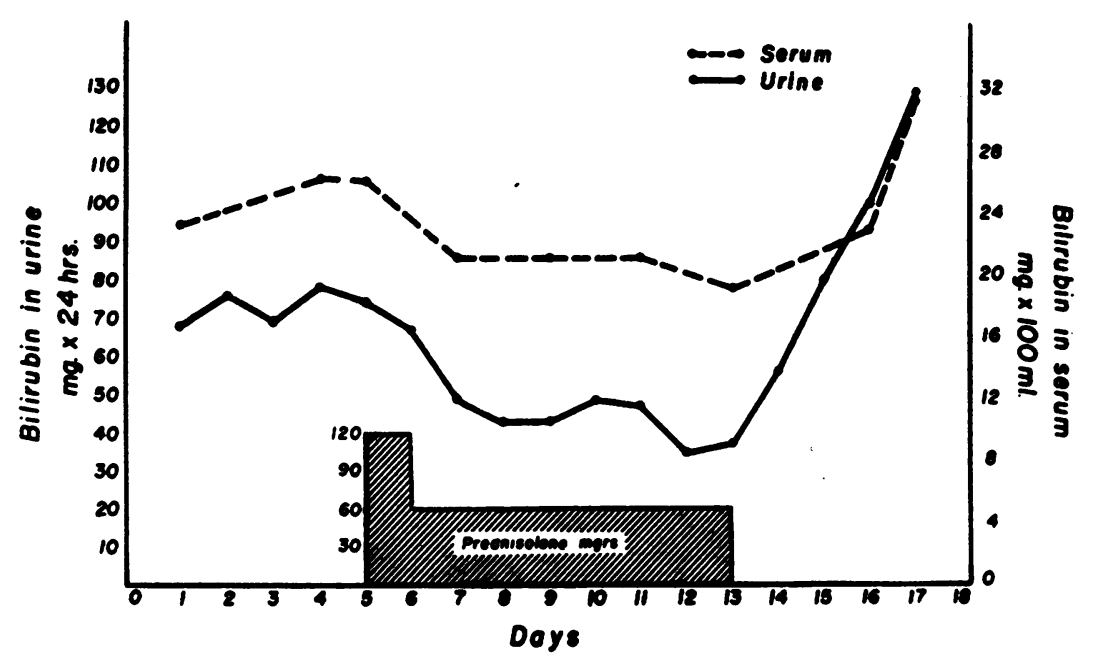

Fig. 5. Influence of Prednisolone on Serum and Urine Bilirubins

Case 5. R.M., 73 year-old female. Hospital number 56/12133.

nemia of post-hepatic jaundice with complete obstruction. The lowering of the serum bilirubin values, although less marked than that observed in acute hepatitis during similar periods, is clearly apparent.

Concomitantly with the lowering of serum bilirubin, a regular decrease of urine bilirubin was observed.

These findings permit the exclusion of an increase in biliary flow or in urinary excretion of bilirubin as the causes for the lowering of hyperbilirubinemia. This could then be explained by a diminution of bilirubinogenesis. The formation of bilirubin could diminish by a slowing down of red cell destruction or of hemoglobin breakdown. The metabolism of hemoglobin could also deviate to colorless compounds which do not react with the diazo reagent. The possibility cannot entirely be ruled out that the effect of the steroids is exerted on the liver, and that a hepatic bilirubinogenesis which is inhibited by them could exist.

The results reported suggest the possibility that the effect of cortisone on bilirubinogenesis may play a role in the rapid drop of serum bilirubin in acute hepatitis.

\section{REFERENCES}

1. Ducci, H., and Motlis, J., Hepatitis fulminante-Recuperación. Rev. Médica de Chile, 1951, 79, 590.

2. Evans, A. S., Sprinz, H., and Nelson, R. S., Adrenal hormone therapy in viral hepatitis. I. The effect of ACTH in the acute disease. Ann. Int. Med., 1953, 38, 1115.

3. Siegenthaler, W., and Suter, L., Zur Behandlung der Hepatitis epidemica mit Cortison. Schweiz. med. Wchnschr., 1955, 85, 1051.

4. Huber, T. E., and Wiley, A. T., Cortisone in the treatment of infectious hepatitis. Ann. Int. Med, 1955, 42, 1011.

5. Solem, J. H., and Olsen, A., The course of icterus index and prothrombin value during corticotropin treatment of acute hepatitis and obstructive jaundice. A preliminary report. Acta med. Scandinav., 1953, 146, 281.

6. Sborov, V. M., Giges, B., Plough, I. C., and Mandel, W., ACTH therapy in acute viral hepatitis. J. Lab. \& Clin. Med., 1954, 43, 48.

7. Johnson, H. C., and Doenges, J. P., Intrahepatic obstructive jaundice (primary cholestasis), a clinicopathologic syndrome of varied etiology: a review with observations of the use of corticotropin as a diagnostic tool. Ann. Int. Med., 1956, 44, 589.

8. Patterson, P. R., Dingman, J. F., Schwachman, H., and Thorn, G. W., Choleretic action of cortisone. New England J. Med., 1954, 251, 502.

9. Chalmers, T. C., Gill, R. J., Jernigan, T. P., Svec, F. A., Jordan, R. S., Waldstein, S. S., and Knowlton, M., Evaluation of a four-day ACTH test in the differential diagnosis of jaundice. Gastroenterology, 1956, 30, 894.

10. Ducci, H., and Watson, C. J., The quantitative determination of the serum bilirubin with special reference to the prompt-reacting and the chloroformsoluble types. J. Lab. \& Clin. Med, 1945, 30, 293.

11. Malloy, H. T., and Evelyn, K. A., The determination of bilirubin with the photoelectric colorimeter. J. Biol. Chem., 1937, 119, 481. 
12. Watson, C. J., Studies on urobilinogen. I. An improved method for the quantitative estimation of urobilinogen in urifie and feces. Am. J. Clin. Path., 1936, 6, 458.

13. Schwartz, S., Sborov, V., and Watson, C. J., Studies of urobilinogen. IV. The quantitative determination of urobilinogen by mearis of the Evelyn photoelectric colorimeter. Am. J. Clin. Path., 1944, I4, 598.

14. Malloy, H. T., and Evelyn, K. A., Qxidation method for bilirubin determination in bile and meconium with the photoelectric colorimeter. J. Biol. Chem., 1938, 122, 597.

15. Jendrassik, L., and Gróf, P.; Verfahren zur photometrischen Bestimmung des Bilirubins im Harn. Biochem. Ztschr., 1938, 296, 71.

16. Watsón, C. J., and Hawkinson, V., Semiquantitative estimation of bilirubin in the urine by means of the barium-strip modification of Harrison's tests. J. Laб. \& Clin. Med., 1946, 31, 914. 